1411 VALIDATING THE AGREEMENT BETWEEN NEWER INFRARED FOREHEAD THERMOMETRY WITH AXILLARY DIGITAL THERMOMETRY IN NEONATES

doi:10.1136/archdischild-2012-302724.1411

'AR Sethi, 'AS Nimbalkar, 'DV Patel, ${ }^{2}$ AG Phatak, ${ }^{1}$ '2 SM Nimbalkar. 'Department of Pediatrics, Pramukhswami Medical College; ${ }^{2}$ Central Research Services, Charutar Arogya Mandal, Anand, India

Background and Aims The traditional mercury thermometer has been replaced by the more "user friendly" digital thermometer. As accuracy is comparable with both instruments and mercury remains an environmental hazard they are no longer recommended. New non invasive method of measuring temperature may reduce infection rate as well as intangible pain and suffering of neonate.

Methods The body temperature of patients admitted in Neonatal Intensive Care Unit was measured using axillary digital thermometer as well as a handheld infrared non touch thermometer. Patients placed under radiant warmers were included. Temperature recordings were taken as required routinely for clinical care. Axillary temperature was recorded within 30 seconds and the forehead temperature within 5 seconds.

Results The body temperature measured by Axillary digital thermometer and forehead method do not agree well (95\% limits of agreement: $-4.2,2.2)$. A trend was observed suggesting that agreement depends on the magnitude of the temperature. The agreement slightly improved when patients in warmer were excluded $(95 \%$ limits of agreement: $-4.1,2.1)$ with similar trend. The best possible agreement was observed between warmer and axillary temperature but was not clinically acceptable (95\% limits of agreement: -0.99 , 2.36).

Conclusion Forehead temperature due to non touch may appear less disturbing to the neonate and also time saving for the nurse but they are misleading. The infrared technology needs further improvement before it can be used in our setting. Although advent of technology is tempting, a scientific validation of new technology under different settings is caveat before adapting it.

\section{TRANSCUTANEOUS BILIRUBINOMETERS USED WITHIN A STRUCTURED PATHWAY PREDICTS HIGH SERUM BILIRUBIN LEVELS IN HEALTHY TERM JAUNDICED NEONATES MONITORED AT HOME}

doi:10.1136/archdischild-2012-302724.1412

'JO Menakaya, 'S Davies, ${ }^{2} \mathrm{~N}$ Callender, Jaundice Early Treatment Study In Term Newborns (JETSET). 'Neonatal Paediatrics; ${ }^{2}$ Obstetrics \& Gynaecology, Hillingdon Hospital NHS Foundation Trust., London, UK

Background UK (NICE) guidelines recommend that bilirubin is monitored in all jaundiced babies. We implemented a home based integrated care pathway to monitor healthy term jaundiced neonates with transcutaneous bilirubinometers $\left(T_{c} B\right)$. Babies were readmitted to hospital for phototherapy at total serum bilirubin $(\mathrm{TsB}) \geq 340 \mu \mathrm{mol} / \mathrm{l}$. TcB $\leq 250 \mu \mathrm{mol} / 1$ correlates highly with TsB.

Aim To determine the $T c B$ values in term babies monitored at home that could predict TsB values $\geq 340 \mu \mathrm{mol} / 1$.

Methods Healthy jaundiced neonates were monitored at home using Bilichek ${ }^{\circledR}$ (Ver 6.12) bilirubinometer. Babies with $\mathrm{TcB}>$ $250 \mu \mathrm{mol} / \mathrm{l}$ had TsB measured using the Beckman Coulter timed endpoint diazo method within 4 hours to confirm result. We carried out statistical analysis of the paired samples to determine the TcB value with the best predictive value for $\mathrm{TsB} \geq 340 \mu \mathrm{mol} / 1$.

Results Eighty-three paired samples were analysed from 63 babies $6(7 \%)$ had TsB values of $\geq 340 \mu \mathrm{mol} / \mathrm{l}$. The Receiver Operating Characteristics (ROC) curve analysis suggested an area under the curve (AUC) of 0.9037 . TcB values $\geq 315 \mu \mathrm{mol} / 1$ predicted $\mathrm{Ts} B \geq 340$ $\mu \mathrm{mol} / \mathrm{l}$ with sensitivity of $0.83(0.36,1.00)$; specificity of $0.82(0.71$,
$0.90)$; positive predictive value of $0.26(0.09,0.51)$ and overall accuracy of $0.82(0.72,0.90)$. TcB values $\geq 303 \mu \mathrm{mol} / 1$ predictive ability had a sensitivity of $1.00(0.54,1.00)$; specificity of $0.71(0.60,0.81)$, positive predictive value of $0.21(0.08,0.41)$ and overall accuracy $0.73(0.63,0.83)$.

Conclusion Bilichek ${ }^{\circledR} \mathrm{TcB}$ of $303 \mu \mathrm{mol} / 1$ had higher sensitivity but lower specificity than TcB of $315 \mu \mathrm{mol} / \mathrm{l}$ for predicting TsB values $\geq 340 \mu \mathrm{mol} / 1$ in healthy term jaundiced neonates monitored at home.

\section{RELATION BETWEEN MATERNAL BLOOD LEVEL OF SOME METALS AND BIRTH WEIGHT USING LIBS}

doi:10.1136/archdischild-2012-302724.1413

\section{J Alsharnoubi. Cairo University, Cairo, Egypt}

Objective Aim of this study is to investigate the relation between intensities of some blood metals as $\mathrm{Fe}, \mathrm{Zn}, \mathrm{Pb}$, and $\mathrm{Cd}$, in both mothers and their newborn, and their effects on the newborn birth weight, height as well as head circumference using Laser Induced Breakdown spectroscopy LIBS as a non invasive technique.

Methods 34 pregnant women and their normal birth weight newborn (NBW, group I) were recruited and matched against 34 pregnant women and their low birth weight newborn (LBW, group II). Blood samples were collected from the umbilical cords of the newborns from both groups and venous blood samples were taken from their mothers after delivery. Samples were prepared then exposed to laser. We used the laser induced breakdown Spectroscopy (LIBS), to analyze the metal intensity of $(\mathrm{Cd}, \mathrm{Pb}, \mathrm{Zn}$, and $\mathrm{Fe})$, for each sample for both mother and her infant.

Results There were significant differences between both groups regarding $\mathrm{Cd}, \mathrm{Pb}, \mathrm{Zn}$, more in group I, while there were no significant differences in both groups in the mother and her newborn regarding to Fe. There were significant differences regarding $\mathrm{Cd}, \mathrm{Pb}$, $\mathrm{Zn}$, more in group I, while no significant differences in both groups in the mother and her newborn regarding to Fe.

Conclusion We found that there was significant negative correlation between birth weight and maternal blood intensity in both groups. We found positive correlation between maternal and newborn blood metals and a negative correlation between intensity of both maternal and infant blood metals regarding weight in both groups.

\section{INSIGHTS INTO NEONATAL ORAL FEEDING PATHOLOGY THROUGH RNA SEQUENCING OF SALIVARY SAMPLES}

doi:10.1136/archdischild-2012-302724.1414

1,2 JL Maron, ${ }^{3} \mathrm{KL}$ Bodi, ${ }^{3} \mathrm{KL}$ Johnson, ${ }^{2,4} \mathrm{DW}$ Bianchi. 'Pediatrics, Floating Hospital for Children, Tufts University; ${ }^{2}$ Mother Infant Research Institute at Tufts Medical Center; ${ }^{3}$ Tufts University School of Medicine: ${ }^{4}$ Pediatrics, Division of Genetics, Boston, MA, USA

Background and Aims To improve our understanding of newborn feeding pathophysiology at the molecular level, our laboratory studies transcripts in neonatal saliva. Previously, we used whole transcriptome microarrays. Here, we tested the hypothesis that sequencing of RNA would provide additional and more specific information.

Methods RNA was extracted and prepared for sequencing from salivary samples $(10 \mu \mathrm{L})$ collected from two term infants matched for post-conceptual age, gender and ethnicity who could and could not orally feed, respectively. Paired-end $100 \times 100$ base pair sequencing was performed on the Illumina HiSeq 2000. Sequence data were aligned against human reference genome GRCH37/hg19. Cuffdiff analysis identified differentially expressed genes, promoters, and splicing variants between subjects. Ingenuity Pathway Analysis was performed on statistically significantly differentially expressed genes. 